\title{
Activation of the AT1R/HIF-1 $\alpha /$ ACE Axis Mediates Angiotensin II-Induced VEGF Synthesis in Mesenchymal Stem Cells
}

\author{
Chao Liu, Jing-Wen Zhang, Liang Hu, Yi-Chen Song, Lu Zhou, Yue Fan, Hong-Yi Zhu, \\ Yu Wang, and Qing-Ping Li
}

Collaborative Innovation Center for Cardiovascular Disease Translational Medicine, Department of Pharmacology, Nanjing Medical University, Nanjing 210029, China

Correspondence should be addressed to Qing-Ping Li; pharm_qpli@njmu.edu.cn

Received 30 June 2014; Revised 16 August 2014; Accepted 17 August 2014; Published 20 October 2014

Academic Editor: Ken-ichi Isobe

Copyright (C) 2014 Chao Liu et al. This is an open access article distributed under the Creative Commons Attribution License, which permits unrestricted use, distribution, and reproduction in any medium, provided the original work is properly cited.

A local renin-angiotensin system (RAS) is expressed in mesenchymal stem cells (MSCs) and regulates stem cell function. The local RAS influences the survival and tissue repairing ability of transplanted stem cells. We have previously reported that angiotensin II (Ang II) pretreatment can significantly increase vascular endothelial growth factor (VEGF) synthesis in MSCs through the ERK1/2 and Akt pathways via the Ang II receptor type 1 (AT1R). However, the role of angiotensin-converting enzyme (ACE) has not been clarified. Furthermore, whether Ang II pretreatment activates hypoxia-inducible factor-1 $\alpha$ (HIF-1 $\alpha$ ) in MSCs has not been elucidated. Our data show that both ACE and HIF- $1 \alpha$ are involved in promoting VEGF expression in MSCs, and that both are upregulated by Ang II stimulation. The upregulation of ACE appeared after the rapid degradation of exogenous Ang II, and led to the formation of endogenous Ang II. On the other hand, the ACE inhibitor, captopril, attenuated Ang II-enhanced HIF-1 $\alpha$ upregulation, while HIF- $1 \alpha$ suppression markedly attenuated ACE expression. This interesting finding suggests an interaction between ACE and HIF- $1 \alpha$. We conclude that Ang II pretreatment, as a trigger, activated the AT1R/HIF-1 $\alpha / \mathrm{ACE}$ axis that then mediated Ang II-induced VEGF synthesis in MSCs.

\section{Introduction}

A local renin-angiotensin system (RAS) has been reported to be expressed in rat mesenchymal stem cells (MSCs). The RAS components, angiotensin II receptors and angiotensinconverting enzyme (ACE), and the de novo synthesis of angiotensin II (Ang II) were detected in MSCs, suggesting that the local RAS could regulate MSC function by an autocrine-paracrine mechanism [1]. MSCs have recently been reported to be suitable for cell-based therapy of ischemic heart disease $[2,3]$. The major mechanism of this therapy is a paracrine action $[4,5]$. Our previous study revealed that Ang II pretreatment increases vascular endothelial growth factor (VEGF) synthesis in MSCs through the ERK1/2 and Akt pathways via the Ang II receptor type 1 (AT1R) [6]. However, the roles of other RAS components and their interactions in MSCs have not been clarified.

$\mathrm{ACE}$ is responsible for converting angiotensin I into Ang II. Numerous studies have demonstrated that ACE is involved in angiogenesis and VEGF expression in different tumor lines
[7-9]. An antiangiogenic effect of ACE inhibitors has been observed in various cancer models, where ACE inhibitors have been shown to attenuate tumor growth and VEGF levels [10-12]. The role of ACE in Ang II-induced VEGF synthesis in MSCs is not known.

Hypoxia-inducible factor- $1 \alpha$ (HIF- $1 \alpha$ ) regulates the expression of VEGF and other proangiogenic genes in response to hypoxia [13]. It is unstable under normoxic conditions and is stabilized under hypoxia [13]. Recent evidence suggests that some nonhypoxic stimuli, such as hormones, growth factors, and cytokines, activate HIF- $1 \alpha$ in a cell-specific manner [14-18]. Therefore, we hypothesized that Ang II may stimulate VEGF expression via HIF-1 $\alpha$ signaling in MSCs. HIF- $1 \alpha$ is the upstream regulator of ACE in hypoxic human pulmonary artery smooth muscle cells (hPASMC) [19]. Whether HIF-1 $\alpha$ influences ACE or other RAS components in MSCs has not been elucidated.

In the present study, we have investigated the roles of ACE and HIF- $1 \alpha$ after Ang II pretreatment and revealed 
the signaling pathway by which ACE and HIF-1 $\alpha$ mediate VEGF secretion in MSCs.

\section{Materials and Methods}

2.1. Ethics Statement. This study was approved by the Committee on the Ethics of Animal Experiments of Nanjing Medical University and complied with the recommendations in the Guide for the Care and Use of Laboratory Animals of the National Institutes of Health. All efforts were made to minimize the number of animals used and their suffering.

2.2. Reagents. Ang II and captopril were purchased from Sigma (St. Louis, MO, USA). Rat VEGF enzyme-linked immunospecific assay (ELISA) kit was from R\&D systems (Minneapolis, MN, USA). Small interfering RNA was obtained from GenePharma (Shanghai, China). The Ang II radioimmunoassay kit (D02PJB) was provided by Beijing North Institute of Biological Technology (Beijing, China). The ACE activity kit was provided by Nanjing Jiancheng Bioengineering Institute (Nanjing, China). Antibodies raised against HIF$1 \alpha$ (Novus Biologicals, USA), ACE (Santa Cruz Biotechnology, USA), were used. Lipofectamine 2000 was purchased from Life Technologies (California, USA). Faststart Universal SYBR Green Master (ROX) was from Roche (Mannheim, Germany).

2.3. Isolation and Culture of MSCs. Male Sprague-Dawley (SD) rats weighing 60-80 g were provided by the Experimental Animal Center of Nanjing Medical University (Nanjing, China). Rats were killed by cervical dislocation. MSCs were generated by flushing the femurs with sterile DMEM (GIBCO, USA) and plated in $75 \mathrm{~cm}^{2}$ primary culture flasks with DMEM containing $10 \%$ fetal bovine serum (FBS, Hyclone, USA) as previously described [20]. Nonadherent cells were removed after $48 \mathrm{~h}$ and the media were replaced every $2 \mathrm{~d}$ for adherent cells. Each primary culture was passaged to new flasks when MSCs grew to approximately $80 \%$ confluence. Cells at passages two to five were used for the experiments.

2.4. Real-Time Quantitative PCR ( $q P C R)$. Total RNA was extracted using the TRIzol reagent (Invitrogen Life Technologies, Gaithersburg, MD) and stored at $-80^{\circ} \mathrm{C}$. The SYBR Green Master was used according to the manufacturer's instructions. The primer sequences (sense/antisense) were as follows: VEGF, $5^{\prime}$-GCGGGCTGCTGCAATG-3' $/ 5^{\prime}$-TGCAACGCGAGTCTGTGTTT-3'; ACE, $5^{\prime}$-ACGGAAGCATCACCAAGGAG-3' $/ 5^{\prime}$-TGGCACATTCGCAGGAACG$3^{\prime} ; \beta$-actin, $5^{\prime}$-GCACCGCAAATGCTTCTA-3 $3^{\prime} / 5^{\prime}$-GGTCTTTACGGATGTCAACG- $3^{\prime}$. The specificity of the amplification product was determined by performing a melting curve analysis. Standard curves were generated for the expression of each gene by using serial dilutions of known quantities of the corresponding cDNA gene template. Relative quantification of the signals was performed by normalizing the signals of different genes with the $\beta$-actin signal.
2.5. Western Blot Analysis. Whole-cell extracts were prepared using lysis buffer (1\% Triton X-100, 20 mM HEPES [pH 7.5], $5 \mathrm{mM} \mathrm{MgCl}_{2}$, 1 mM EDTA, 1 mM EGTA, 1 mM dithiothreitol, $1 \mathrm{mM}$ phenylmethylsulphonyl fluoride, and $1 \mathrm{mg} / \mathrm{mL}$ each of leupeptin, aprotinin, and pepstatin). Protein samples were separated on a $10 \%$ SDS-PAGE gel and transferred to a nitrocellulose membrane. The membrane was blocked in 5\% milk/TBST and incubated overnight at $4^{\circ} \mathrm{C}$ with the following primary antibodies: ACE, HIF- $1 \alpha$, and $\beta$-actin. Membranes were exposed to HRP-conjugated secondary antibodies for $2 \mathrm{~h}$ at room temperature and protein expression was detected using enhanced chemiluminescence (ECL) detection reagent (Pierce).

2.6. ELISA Analysis. The MSC supernatants from all experimental groups were collected, centrifuged to remove cell debris, and stored at $-80^{\circ} \mathrm{C}$ for analysis. VEGF concentration was measured according to the instructions of the ELISA kit. Spectrophotometric evaluation of VEGF levels was made using a Synergy HT multidetection microplate reader (BioTek).

2.7. siRNA Transfection. MSCs were seeded in 6-well plates. After 1 day, the cells were transfected with siRNA (100 nmol/ L) using Lipofectamine 2000 according to the manufacturer's instructions. A corresponding random siRNA sequence was used as a negative control (Control). The siRNA sequences (sense/antisense) used were as follows: HIF-1 $\alpha$ siRNA: $5^{\prime}$ GAGCUCCCAUCUUGAUAAATT- $3^{\prime} / 5^{\prime}$-UUUAUCAAGAUGGGAGCUCTT-3'; Control: $5^{\prime}$-UUCUCCGAACGUGUCACGUTT- $3^{\prime} / 5^{\prime}$-ACGUGACACGUUCGGAGAATT$3^{\prime}$. The effect of siRNA transfection was detected by western blot. Forty-eight hours after transfection, the cells were incubated in Ang II (100 nM) and collected in preparation for experiments.

2.8. Radioimmunoassay. The MSCs were seeded in 6-well plates and treated with $100 \mathrm{nM}$ Ang II. The supernatants of pretreated MSCs were collected at different time points within $33 \mathrm{~h}$ and stored at $-80^{\circ} \mathrm{C}$ for analysis. Supernatants of untreated MSCs were also collected. Ang II concentration was measured according to the radioimmunoassay kit instructions.

2.9. ACE Activity Measurement. ACE activity was measured according to the kit instructions. In brief, ACE activity was determined with an artificial substrate $\mathrm{N}$-[3-(2furyl)acryloyl]-L-phenylalanylglycylglycine (FAPGG) in a reaction mixture containing $25 \mu \mathrm{L}$ sample of MSC culture media and $100 \mu \mathrm{L} 2.5 \mathrm{mM}$ FAPGG. Measurements were performed in 96 -well plates at $37^{\circ} \mathrm{C}$. Changes in optical density $(340 \mathrm{~nm})$ were measured at 5-minute intervals with a plate reader (BioTek). ACE activity was calculated according to the observed decrease in optical density.

2.10. Statistical Analysis. All data were expressed as mean \pm SEM. Student's $t$-test was used for two-group comparisons and one-way ANOVA followed by Bonferroni correction was used for multiple group comparisons. A value of $P<0.05$ was considered to be statistically significant. 


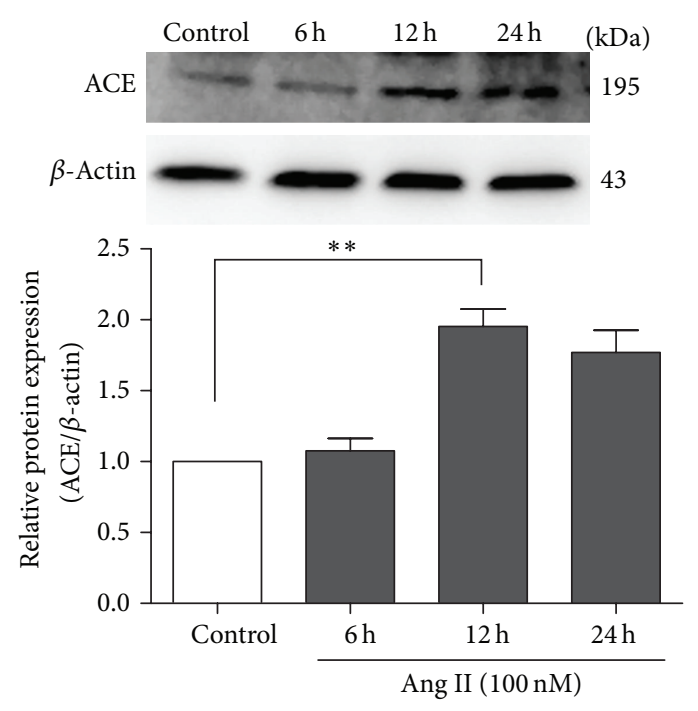

(a)

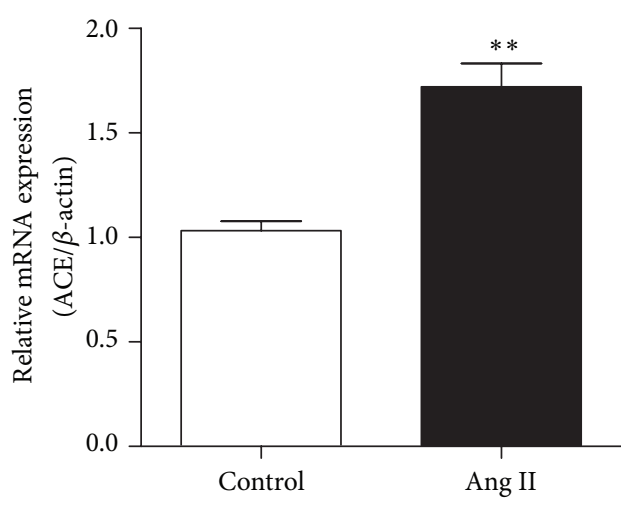

(c)

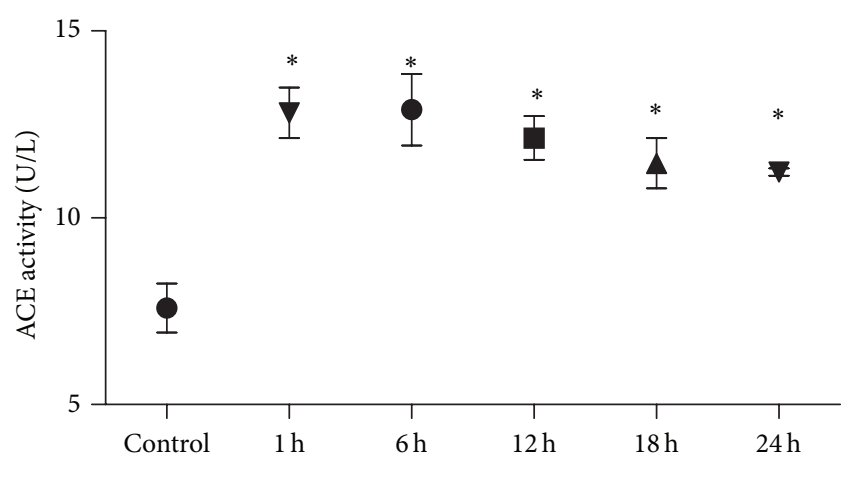

(b)

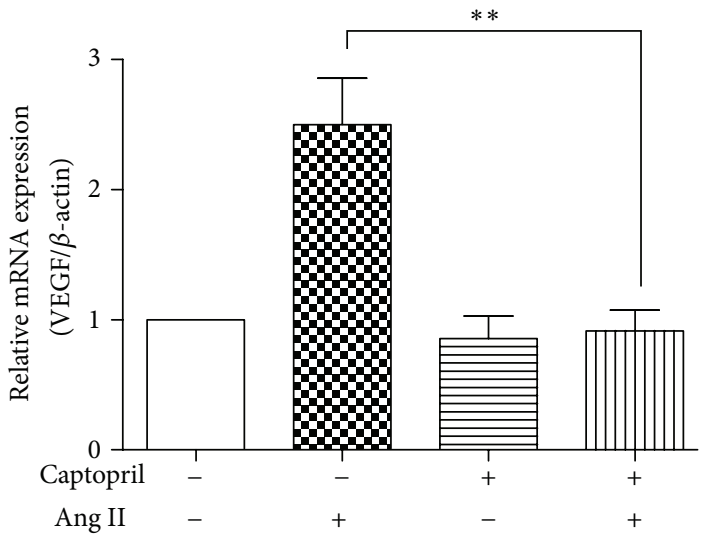

(d)

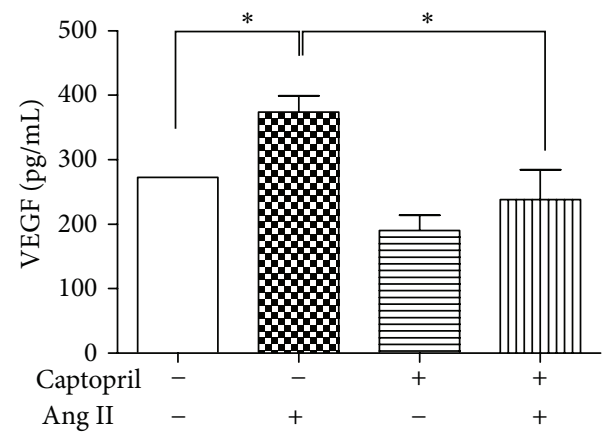

(e)

FIGURE 1: Upregulation of ACE after Ang II-induced VEGF synthesis. (a) Western blot analysis of ACE. MSCs were exposed to Ang II (100 nM) for $6 \mathrm{~h}, 12 \mathrm{~h}$, or $24 \mathrm{~h} . n=5,{ }^{* *} P<0.01$. (b) Determination of ACE activity. MSCs were exposed to Ang II (100 nM) for $1 \mathrm{~h}, 6 \mathrm{~h}, 12 \mathrm{~h}, 18 \mathrm{~h}$, or 24 h. $n=5,{ }^{*} P<0.05$ every other group versus control. (c) Real-time qPCR examination of ACE mRNA levels. MSCs were exposed to Ang II (100 nM) for 12 h. $n=5,{ }^{* *} P<0.01$. (d) Real-time qPCR analysis of VEGF mRNA level, ${ }^{* *} P<0.01$. (e) ELISA of VEGF secretion. MSCs were pretreated with or without captopril $(1 \mu \mathrm{M}$ for $1 \mathrm{~h})$ before exposure to Ang II $(100 \mathrm{nM})$ for $12 \mathrm{~h} . n=5,{ }^{*} P<0.05$.

\section{Results}

3.1. ACE Was Upregulated by Ang II Stimulation and Involved in VEGF Expression. To determine the influence of Ang II stimulation on ACE, we examined ACE mRNA and protein expression. After exposure to $100 \mathrm{nM}$ Ang II, ACE protein expression in MSCs doubled within $24 \mathrm{~h}$ in a time-dependent manner $(P<0.01$; Figure 1(a)). Real-time qPCR showed that the ACE mRNA level in pretreated MSCs increased to over 1.5 -fold of control $(P<0.01$; Figure $1(\mathrm{c}))$. Additionally, 


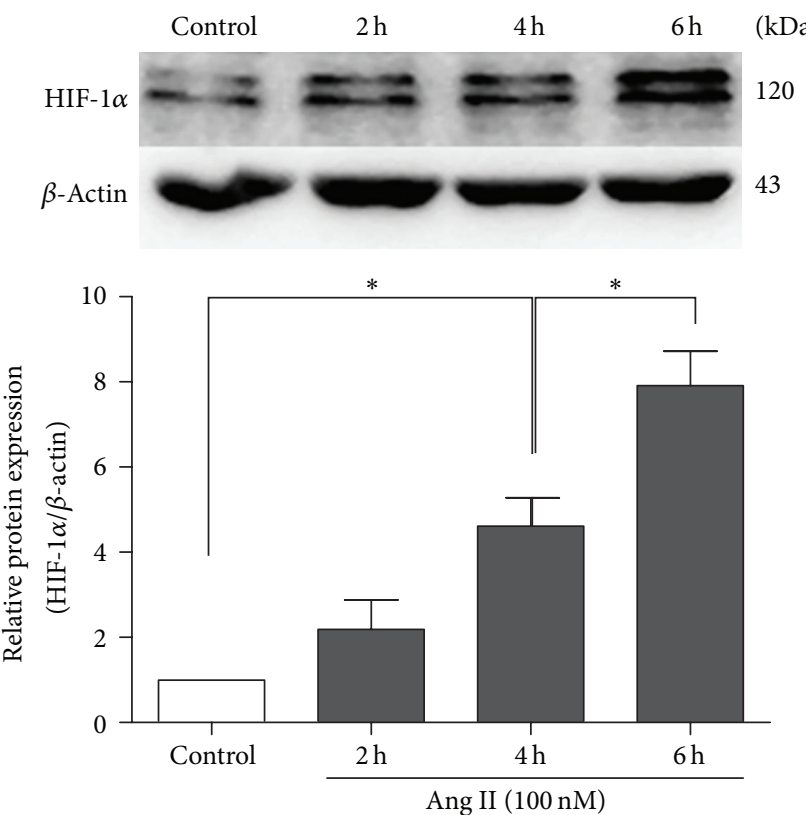

(a)

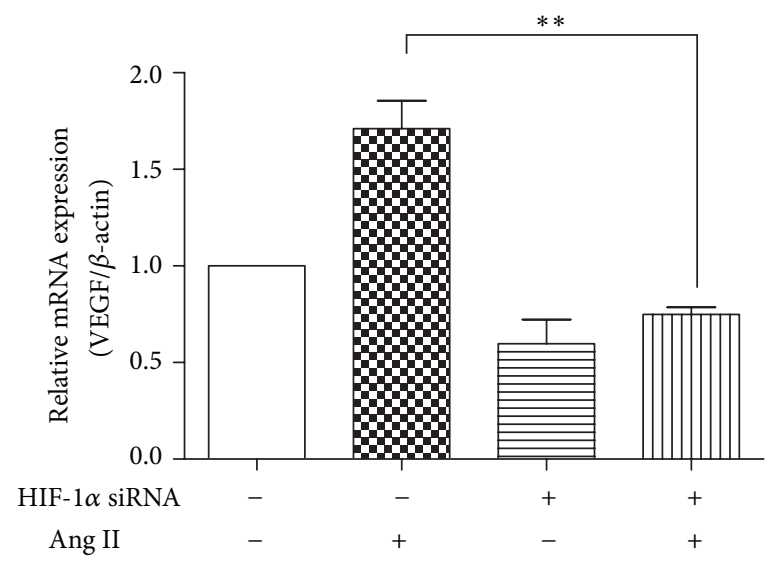

(c)
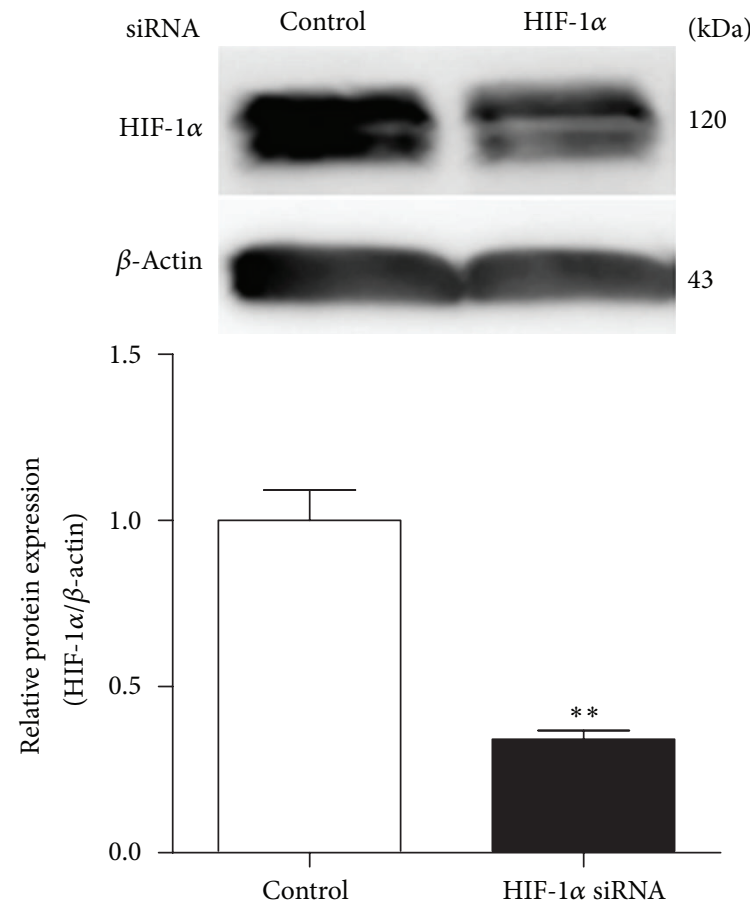

(b)

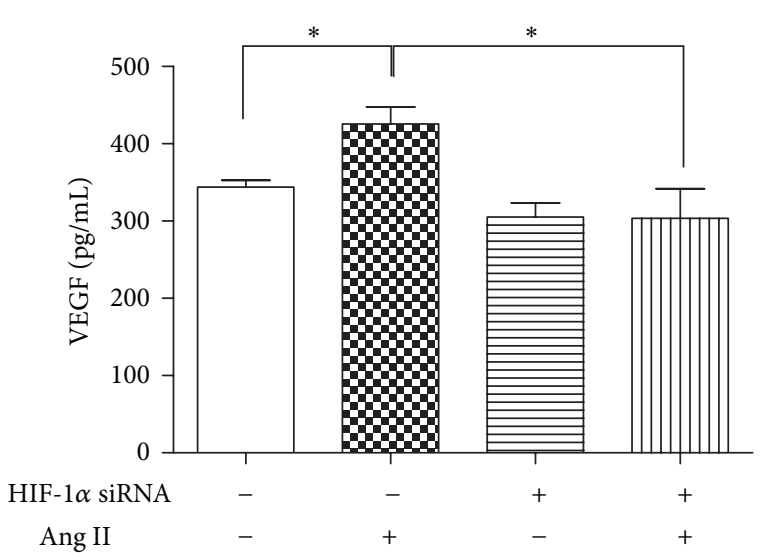

(d)

FIGURE 2: Activation of HIF-1 $\alpha$ after Ang II-stimulated VEGF synthesis. (a) Western blot analysis of HIF-1 $\alpha$ expression. MSCs were exposed to Ang II $(100 \mathrm{nM})$ for $2 \mathrm{~h}, 4 \mathrm{~h}$, or $6 \mathrm{~h} . n=5,{ }^{*} \mathrm{P}<0.05$. (b) Western blot analysis of HIF-1 $\alpha$ siRNA efficiency. $n=3,{ }^{* *} P<0.01$. (c) Real-time qPCR of VEGF mRNA level, ${ }^{* *} P<0.01$. (d) ELISA of VEGF secretion. MSCs were pretreated by HIF-1 $\alpha$ siRNA before being exposed to Ang II $(100 \mathrm{nM})$ for $12 \mathrm{~h} . n=5 .{ }^{*} P<0.05$.

Ang II stimulation induced 1.7-fold increase in ACE activity $(P<0.05$; Figure 1(b)). To ascertain whether ACE was involved in Ang II-induced VEGF synthesis, MSCs were preincubated with $1 \mu \mathrm{M}$ captopril, a specific inhibitor of ACE, for $1 \mathrm{~h}$ before treatment with $100 \mathrm{nM}$ Ang II for $12 \mathrm{~h}$. VEGF mRNA expression and VEGF synthesis were then measured. After Ang II stimulation VEGF mRNA expression doubled. This effect was abolished by captopril $(P<0.01$; Figure $1(\mathrm{~d}))$. The Ang II-induced increase of VEGF secretion was also diminished in the captopril preincubation group $(P<0.05$;
Figure 1(e)). Therefore, we conclude that ACE promotes Ang II-induced VEGF expression in MSCs.

3.2. HIF-1 $\alpha$ Was Upregulated by Ang II Stimulation and Involved in VEGF Expression. To test the influence of Ang II stimulation on HIF- $1 \alpha$, we assessed HIF- $1 \alpha$ protein expression after MSCs were exposed to $100 \mathrm{nM}$ Ang II for 2, 4, or $6 \mathrm{~h}$. HIF- $1 \alpha$ was upregulated in a time-dependent manner $(P<0.05$, Figure $2(\mathrm{a}))$. To determine whether the increase of VEGF was mediated by HIF-1 $\alpha$, MSCs were transfected 


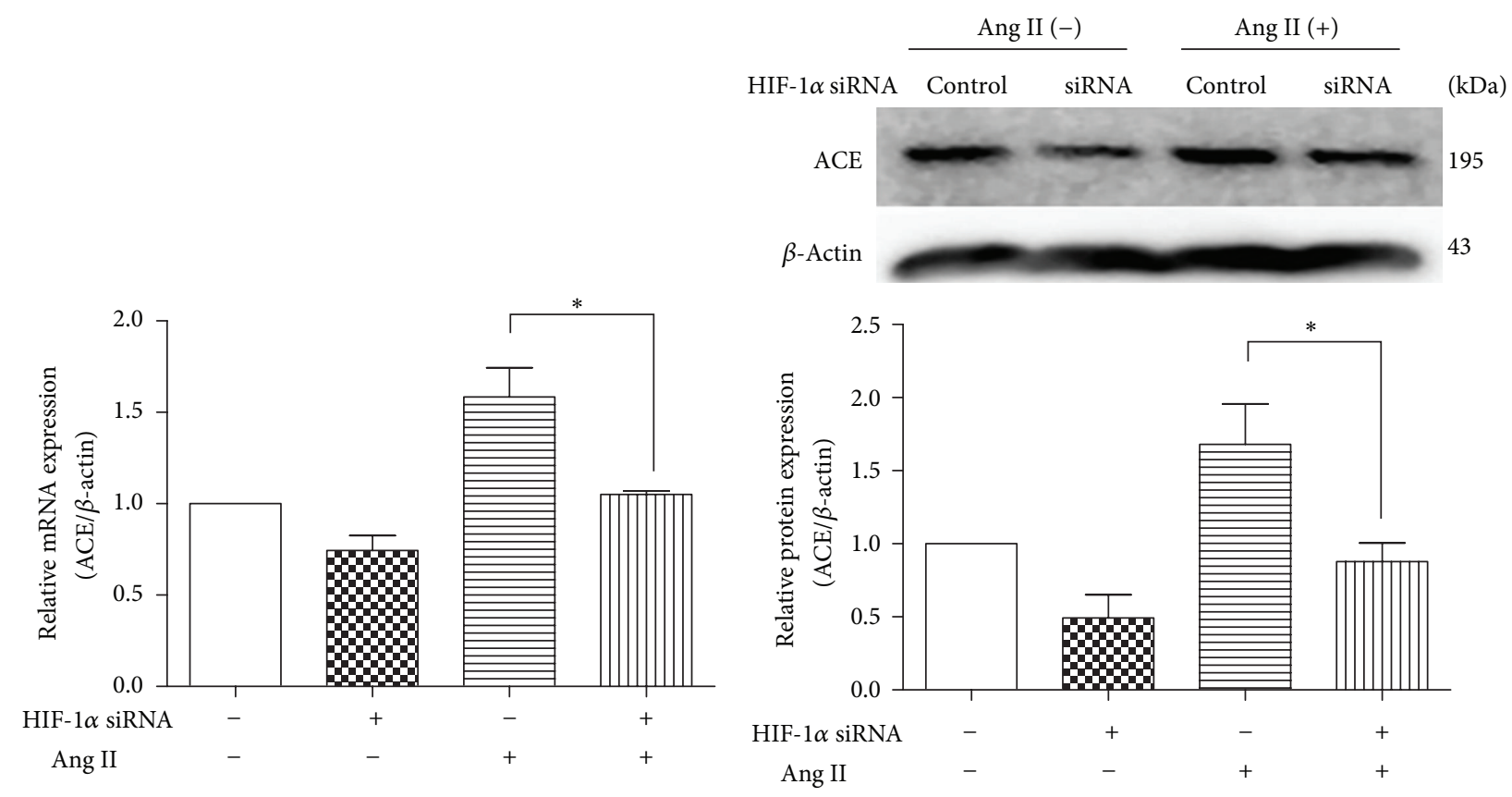

(a)

(b)
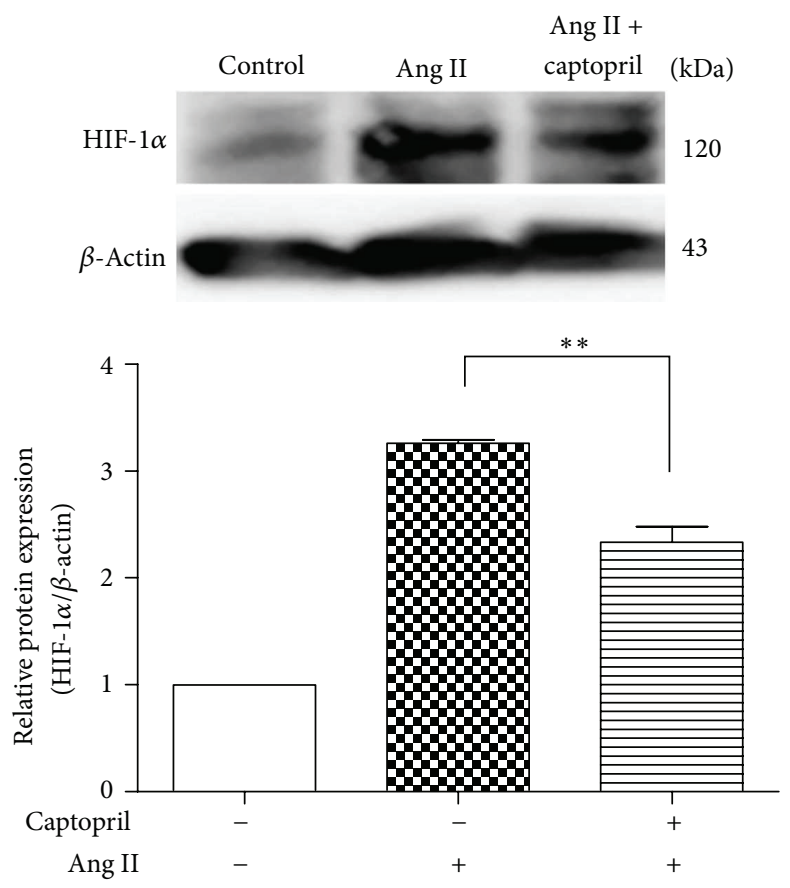

(c)

FIGURE 3: Interaction of HIF- $1 \alpha$ with ACE in MSCs. (a) Real-time qPCR analysis of ACE mRNA level. MSCs were pretreated by HIF-1 $\alpha$ siRNA before being exposed to Ang II (100 nM) for $12 \mathrm{~h} . n=5,{ }^{*} P<0.05$. (b) Western blot analysis of ACE expression. MSCs were pretreated by HIF-1 $\alpha$ siRNA before being exposed to Ang II (100 nM) for 6 h. $n=5,{ }^{*} P<0.05$. (c) Western blot analysis of HIF- $1 \alpha$. MSCs were pretreated with or without captopril $(1 \mu \mathrm{M})$ for $1 \mathrm{~h}$ before they were exposed to Ang II $(100 \mathrm{nM})$ for $12 \mathrm{~h} . n=5,{ }^{* *} P<0.01$.

with HIF-1 $\alpha$ siRNA. The transfection group expressed less HIF- $1 \alpha$ than the control group (Figure 2(b)). The Ang IIinduced VEGF mRNA expression and protein secretion were markedly attenuated by knockdown of HIF- $1 \alpha(P<0.01$, Figure 2(c); $P<0.05$, Figure 2(d)). These results indicate that Ang II promotes VEGF expression via HIF- $1 \alpha$ activation.
3.3. Interactions of HIF-1 $\alpha$ and ACE Were Triggered by Exogenous Ang II. To study the potential interaction of HIF- $1 \alpha$ with ACE, we inhibited HIF-1 $\alpha$ by siRNA and ACE with captopril. Protein expression was analyzed by western blot. Transfection of MSCs with HIF- $1 \alpha$ siRNA resulted in a significant decrease of ACE mRNA and protein expression $(P<0.05$, Figure 3(a); 


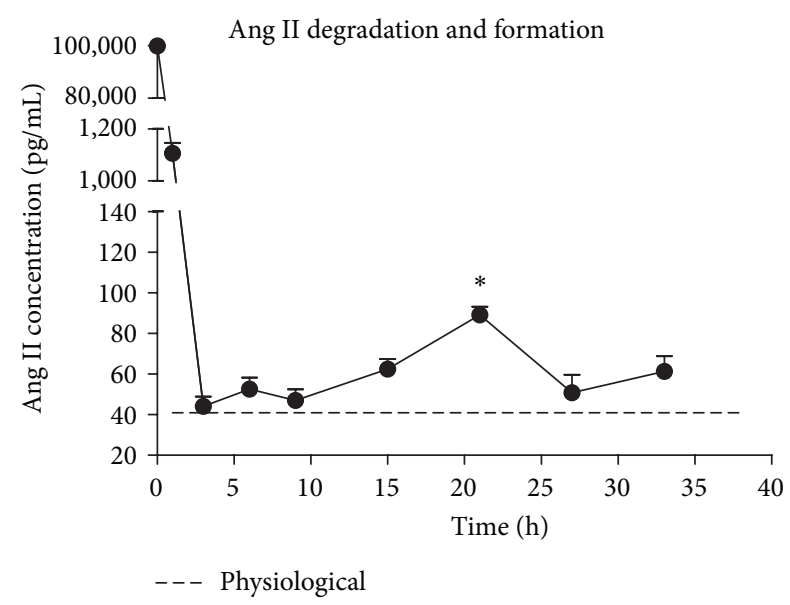

FIgURE 4: Ang II degradation and formation. Ang II concentration was determined by radioimmunoassay. Exogenous Ang II (100 nM) was added to the culture medium at time 0 . Concentrations were determined at $1,3,6,9,15,21,27$, and $33 \mathrm{~h} . n=3 .{ }^{*} P<0.05,21 \mathrm{~h}$ versus 3, 6, 9, $15 \mathrm{~h}$. The concentration of Ang II in untreated MSC culture medium was also tested as the physiological concentration. $n=3$.

$P<0.05$, Figure 3(b)). Interestingly, a downregulation of HIF-1 $\alpha$ protein expression was also observed when MSCs were pretreated with the ACE inhibitor, captopril $(P<$ 0.01 , Figure 3(c)). These results suggest that ACE and HIF$1 \alpha$ might interact with each other during their upregulation after MSCs are stimulated by Ang II.

3.4. Endogenous Ang II Was Augmented following ACE Upregulation after Pretreatment. The cleavage of exogenously added Ang II and the formation of endogenous Ang II in MSC culture media were determined by radioimmunoassay. Exogenous Ang II (100 nM) was added. The Ang II concentration was reduced to $1106 \pm 40.48 \mathrm{pM}$ after $1 \mathrm{~h}$ and continued to drop to $42.45 \pm 4.26 \mathrm{pM}$ after $3 \mathrm{~h}$ (Figure 4 ). However, the Ang II concentration increased significantly from $4 \mathrm{~h}(42.45 \pm 4.26 \mathrm{pM})$ to $21 \mathrm{~h}(89.12 \pm 4.02 \mathrm{pM})$ and then decreased at $27 \mathrm{~h}(50.78 \pm 8.90 \mathrm{pM})$. The Ang II concentration tended to increase again from 28 to $33 \mathrm{~h}$. The Ang II level of untreated MSCs, that is, the physiological concentration, was $41.05 \pm 2.83 \mathrm{pM}$ (Figure 4 ).

\section{Discussion}

The main findings of this study are that ACE and HIF- $1 \alpha$ mediate Ang II-induced VEGF synthesis in MSCs and that ACE and HIF- $1 \alpha$ appear to interact in this process. Both ACE and HIF- $1 \alpha$ mediate the increase of VEGF synthesis by forming a positive feedback of the AT1R/HIF- $1 \alpha / \mathrm{ACE}$ axis.

We have reported previously that Ang II stimulation could increase VEGF synthesis in MSCs through the ERK1/2 and Akt pathways via the AT1R [6]. This study examined the pathway that regulates ACE following activation of the AT1R by Ang II. Here, we report for the first time that ACE is upregulated after Ang II stimulation, and ACE upregulation also increases VEGF synthesis in MSCs (Figure 1). The upregulation of ACE appeared after the rapid degradation of exogenous Ang II and led to the formation of endogenous Ang II (Figure 4). Endogenous Ang II would be expected to continue to exert biological effects. We suggest that exogenous Ang II, as a trigger, induces VEGF synthesis with the formation of an Ang II-AT1R-ACE-VEGF autocrine system (Figure 5). In this process, exogenous Ang II is rapidly degraded. Neprilysin, an endopeptidase expressed on the cell surface, is the main enzyme responsible for degrading Ang II in preadipocytes and adipocytes [21]. Given the high activity of neprilysin in MSCs [22, 23], we attribute the rapid degradation of exogenous Ang II to neprilysin. A local RAS in MSCs was discovered 10 years ago [1]. The RAS components, angiotensinogen, renin, ACE, and AT receptors, were found to be present in cultured MSCs. The de novo synthesis of Ang II by MSCs was also detected. These findings demonstrated that a potential autocrine-paracrine mechanism existed in the local RAS of MSCs [1]. In this study, we also revealed that ACE activity of MSCs was raised by Ang II stimulation (Figure 1). Ang II concentration in culture media of MSCs increased from $4 \mathrm{~h}$ to $21 \mathrm{~h}$ (Figure 4 ), which was a result of the balance between ACE and neprilysin activities. In summary, a transient high level of exogenous Ang II acts as a trigger to upregulate ACE and induces a positive feedback of the RAS in MSCs. Although Schunkert et al. [24] reported Ang II infusion decreased ACE mRNA levels in the lung and testis of rats, later studies have confirmed a positive feedback effect on the local RAS with an ACE/AT1R-dependent mechanism in the kidney $[25,26]$.

HIF- $1 \alpha$ is known to regulate VEGF expression in hypoxic conditions or after chemical stimulation. Several studies have indicated that HIF- $1 \alpha$ can also be activated by Ang II $[17,27,28]$. In hPASMC, the phosphatidylinositol 3kinase (PI3 K)/Akt pathway may mediate Ang II-stimulated HIF-1 $\alpha$ upregulation [29]. However, the influence of Ang II pretreatment on HIF-1 $\alpha$ signaling in MSCs has not been previously reported. In this study, we found for the first time that HIF- $1 \alpha$ could be upregulated by Ang II stimulation and that the inhibition of HIF-1 $\alpha$ significantly blocked VEGF enhancement by Ang II stimulation in MSCs (Figure 2). These findings suggest that HIF- $1 \alpha$ is involved in Ang IIinduced VEGF synthesis in MSCs.

To investigate the interaction of HIF- $1 \alpha$ with ACE, we targeted siRNA to HIF- $1 \alpha$ to inhibit its function and found that the inhibition of HIF-1 $\alpha$ significantly blocked ACE upregulation (Figure 3). This finding indicates that HIF-1 $\alpha$ acts as a mediator to promote ACE expression in MSCs. Additionally, when ACE was inhibited by captopril, Ang II-enhanced HIF$1 \alpha$ expression was significantly attenuated (Figure 3 ). This indicates that there is an interaction between HIF- $1 \alpha$ and ACE-Ang II-AT1R signaling in MSCs. As shown in Figure 5, the data suggest that exogenous Ang II upregulates HIF-1 $\alpha$ via the AT1R. Subsequently, HIF- $1 \alpha$ not only activates VEGF expression, but also upregulates ACE expression which then increases endogenous Ang II formation and enhances AT1R activation.

Additionally, we have previously reported that Ang IIinduced STAT3 phosphorylation activates VEGF mRNA 


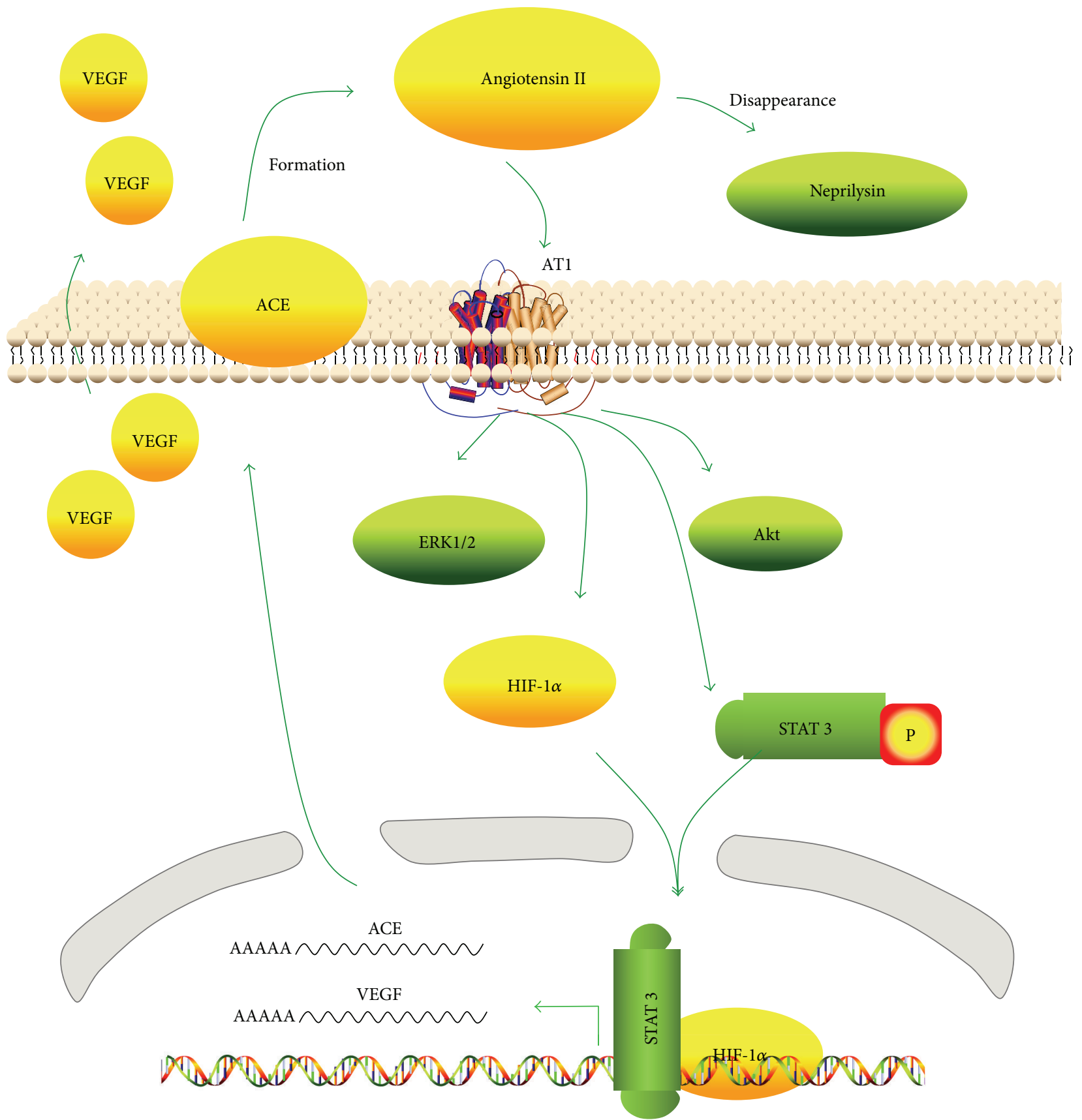

FIgURE 5: Scheme of Ang II-driven VEGF synthesis. Ang II induction sensing via HIF-1 $\alpha$ or STAT3 activates an autocrine loop of ACE upregulation, Ang II formation, and signaling via AT1R, which exerts a positive feedback and further fosters VEGF synthesis in MSCs.

expression and protein synthesis in MSCs [30]. An ACE inhibitor attenuated Ang II-induced STAT3 activation, while STAT3 inhibition diminished the upregulation of ACE. Considering these results, it is reasonable to speculate that the role of STAT3 is similar to that of HIF- $1 \alpha$ in the fact that it not only influences VEGF mRNA expression but also activates ACEAng II-AT1R signaling. We have not examined the interaction between STAT3 and HIF- $1 \alpha$ in MSCs. STAT3 is required for HIF- $1 \alpha$ activation in human renal carcinoma cells and in female MSCs [31-33]. It can be inferred that STAT3 influences the AT1R-HIF- $1 \alpha$-ACE axis either by activating HIF- $1 \alpha$ or by directly upregulating ACE, leading to increased VEGF expression (Figure 5).

In conclusion, the present results show that ACE and HIF- $1 \alpha$ activation are involved in Ang II-induced upregulation of VEGF in MSCs. ACE and HIF- $1 \alpha$ are involved in positive feedback of an AT1R-HIF-1 $\alpha$-ACE-Ang II loop. HIF$1 \alpha$ is possibly activated by STAT3 phosphorylation that is reported to cooperatively foster VEGF synthesis. These data clarify not only the mechanism of the Ang II stimulatory effect but also the mechanisms underlying Ang II-induced VEGF synthesis in MSCs. Our research sheds light on 
the cellular signaling underlying Ang II pretreatment for MSC transplantation. Further research in this area is underway. Under hypoxic conditions to mimic the infracted border-zone of the myocardium, the influence and mechanisms of pathologically elevated Ang II on the survival and paracrine effects of transplanted MSCs are under investigation. Also, we have preliminary results showing that transplantation of Ang II-pretreated MSCs resulted in better cardiac function than untreated MSCs.

\section{Conflict of Interests}

No conflict of interest exists in the submission of this paper. All the authors listed have approved the paper for publication.

\section{Authors' Contribution}

Chao Liu and Jing-Wen Zhang contributed equally to this study.

\section{Acknowledgments}

This study was supported by the National Science Foundation of China (nos. 30973534 and 81173052) and the Jiangsu College Graduate Research and Innovation Program (no. CXZZ13_0604). The funders had no role in study design, data collection and analysis, decision to publish, or preparation of the paper.

\section{References}

[1] W. B. Strawn, R. S. Richmond, E. A. Tallant, P. E. Gallagher, and C. M. Ferrario, "Renin-angiotensin system expression in rat bone marrow haematopoietic and stromal cells," British Journal of Haematology, vol. 126, no. 1, pp. 120-126, 2004.

[2] V. Schächinger, B. Assmus, M. B. Britten et al., "Transplantation of progenitor cells and regeneration enhancement in acute myocardial infarction: final one-year results of the TOPCAREAMI trial," Journal of the American College of Cardiology, vol. 44, no. 8, pp. 1690-1699, 2004.

[3] N. Nagaya, K. Kangawa, T. Itoh et al., "Transplantation of mesenchymal stem cells improves cardiac function in a rat model of dilated cardiomyopathy," Circulation, vol. 112, no. 8, pp. 1128-1135, 2005.

[4] R. Uemura, M. Xu, N. Ahmad, and M. Ashraf, "Bone marrow stem cells prevent left ventricular remodeling of ischemic heart through paracrine signaling," Circulation Research, vol. 98, no. 11, pp. 1414-1421, 2006.

[5] M. Gnecchi, H. He, N. Noiseux et al., "Evidence supporting paracrine hypothesis for Akt-modified mesenchymal stem cellmediated cardiac protection and functional improvement," The FASEB Journal, vol. 20, no. 6, pp. 661-669, 2006.

[6] R.-Z. Shi, J.-C. Wang, S.-H. Huang, X.-J. Wang, and Q.-P. Li, "Angiotensin II induces vascular endothelial growth factor synthesis in mesenchymal stem cells," Experimental Cell Research, vol. 315, pp. 10-15, 2009.

[7] R. Yasumatsu, T. Nakashima, M. Masuda et al., "Effects of the angiotensin-I converting enzyme inhibitor perindopril on tumor growth and angiogenesis in head and neck squamous cell carcinoma cells," Journal of Cancer Research and Clinical Oncology, vol. 130, no. 10, pp. 567-573, 2004.

[8] O. V. Volpert, W. F. Ward, M. W. Lingen et al., "Captopril inhibits angiogenesis and slows the growth of experimental tumors in rats," Journal of Clinical Investigation, vol. 98, no. 3, pp. 671-679, 1996.

[9] H. Yoshiji, S. Kuriyama, M. Kawata et al., "The angiotensinI-converting enzyme inhibitor perindopril suppresses tumor growth and angiogenesis: possible role of the vascular endothelial growth factor," Clinical Cancer Research, vol. 7, no. 4, pp. 1073-1078, 2001.

[10] S.-I. Hii, D. L. Nicol, D. C. Gotley, L. C. Thompson, M. K. Green, and J. R. Jonsson, "Captopril inhibits tumour growth in a xenograft model of human renal cell carcinoma," British Journal of Cancer, vol. 77, no. 6, pp. 880-883, 1998.

[11] H. A. Arafat, Q. Gong, G. Chipitsyna, A. Rizvi, C. T. Saa, and C. J. Yeo, "Antihypertensives as novel antineoplastics: angiotensin-I-converting enzyme inhibitors and angiotensin II type 1 receptor blockers in pancreatic ductal adenocarcinoma," Journal of the American College of Surgeons, vol. 204, no. 5, pp. 996-1005, 2007.

[12] H. Yoshiji, S. Kuriyama, and H. Fukui, "Angiotensin-I-converting enzyme inhibitors may be an alternative anti-angiogenic strategy in the treatment of liver fibrosis and hepatocellular carcinoma: possible role of vascular endothelial growth factor," Tumor Biology, vol. 23, no. 6, pp. 348-356, 2002.

[13] C. W. Pugh and P. J. Ratcliffe, "Regulation of angiogenesis by hypoxia: role of the HIF system," Nature Medicine, vol. 9, no. 6, pp. 677-684, 2003.

[14] R. Fukuda, K. Hirota, F. Fan, Y. D. Jung, L. M. Ellis, and G. L. Semenza, "Insulin-like growth factor 1 induces hypoxiainducible factor 1-mediated vascular endothelial growth factor expression, which is dependent on MAP kinase and phosphatidylinositol 3-kinase signaling in colon cancer cells," The Journal of Biological Chemistry, vol. 277, no. 41, pp. 38205-38211, 2002.

[15] R. Fukuda, B. Kelly, and G. L. Semenza, "Vascular endothelial growth factor gene expression in colon cancer cells exposed to prostaglandin E2 is mediated by hypoxia-inducible factor 1," Cancer Research, vol. 63, no. 9, pp. 2330-2334, 2003.

[16] G. S. Erwin, P. R. Crisostomo, Y. Wang et al., "Estradiol-treated mesenchymal stem cells improve myocardial recovery after ischemia," Journal of Surgical Research, vol. 152, no. 2, pp. 319324, 2009.

[17] D. E. Richard, E. Berra, and J. Pouyssegur, "Nonhypoxic pathway mediates the induction of hypoxia-inducible factor $1 \alpha$ in vascular smooth muscle cells," The Journal of Biological Chemistry, vol. 275, no. 35, pp. 26765-26771, 2000.

[18] A. A. Kazi and R. D. Koos, "Estrogen-induced activation of hypoxia-inducible factor- $1 \alpha$, vascular endothelial growth factor expression, and edema in the uterus are mediated by the phosphatidylinositol 3-kinase/Akt pathway," Endocrinology, vol. 148, no. 5, pp. 2363-2374, 2007.

[19] R. Zhang, Y. Wu, M. Zhao et al., "Role of HIF-1 $\alpha$ in the regulation ACE and ACE2 expression in hypoxic human pulmonary artery smooth muscle cells," The American Journal of Physiology-Lung Cellular and Molecular Physiology, vol. 297, no. 4, pp. L631-L640, 2009.

[20] J. Y. Hahn, H. J. Cho, H. J. Kang et al., "Pre-treatment of mesenchymal stem cells with a combination of growth factors enhances gap junction formation, cytoprotective effect on cardiomyocytes, and therapeutic efficacy for myocardial 
infarction," Journal of the American College of Cardiology, vol. 51, no. 9, pp. 933-943, 2008.

[21] P. Schling and T. Schäfer, "Human adipose tissue cells keep tight control on the angiotensin II levels in their vicinity," The Journal of Biological Chemistry, vol. 277, no. 50, pp. 48066-48075, 2002.

[22] M.-S. Tsai, S.-M. Hwang, K.-D. Chen et al., "Functional network analysis of the transcriptomes of mesenchymal stem cells derived from amniotic fluid, amniotic membrane, cord blood, and bone marrow," Stem Cells, vol. 25, no. 10, pp. 2511-2523, 2007.

[23] T. Katsuda, R. Tsuchiya, N. Kosaka et al., "Human adipose tissue-derived mesenchymal stem cells secrete functional neprilysin-bound exosomes," Scientific Reports, vol. 3, article no. $1197,2013$.

[24] H. Schunkert, J. R. Ingelfinger, A. T. Hirsch et al., "Feedback regulation of angiotensin converting enzyme activity and mRNA levels by angiotensin II," Circulation Research, vol. 72, no. 2, pp. 312-318, 1993.

[25] J. Sadjadi, G. L. Kramer, C.-H. Yu, M. B. Welborn III, and J. G. Modrall, "Angiotensin II exerts positive feedback on the intrarenal renin-angiotensin system by an angiotensin converting enzyme-dependent mechanism," Journal of Surgical Research, vol. 129, no. 2, pp. 272-277, 2005.

[26] L.-X. Zou, J. D. Imig, A. M. von Thun, A. Hymel, H. Ono, and L. G. Navar, "Receptor-mediated intrarenal angiotensin II augmentation in angiotensin II-infused rats," Hypertension, vol. 28, no. 4, pp. 669-677, 1996.

[27] E. L. Pagé, G. A. Robitaille, J. Pouysségur, and D. E. Richard, "Induction of hypoxia-inducible factor-lalpha by transcriptional and translational mechanisms," The Journal of Biological Chemistry, vol. 277, pp. 48403-48409, 2002.

[28] E. Sánchez-López, A. F. López, V. Esteban et al., "Angiotensin II regulates vascular endothelial growth factor via hypoxiainducible factor- $1 \alpha$ induction and redox mechanisms in the kidney," Antioxidants and Redox Signaling, vol. 7, no. 9-10, pp. 1275-1284, 2005.

[29] S. Krick, J. Hänze, B. Eul et al., "Hypoxia-driven proliferation of human pulmonary artery fibroblasts: cross-talk between HIF$1 \alpha$ and an autocrine angiotensin system," The FASEB Journal, vol. 19, no. 7, pp. 857-859, 2005.

[30] J. Zhang, L. Hu, C. Liu, L. Wang, and Q. Li, "Involvement of signal transducer and activator of transcription 3 in angiotensin II-upregulated vascular endothelial growth factor production in mesenchymal stem cells," Acta Universitatis Medicinalis Nanjing (Natural Science), vol. 32, pp. 891-895, 2012.

[31] M. Wang, J. Tan, A. Coffey, J. Fehrenbacher, B. R. Weil, and D. R. Meldrum, "Signal transducer and activator of transcription 3-stimulated hypoxia inducible factor- $1 \alpha$ mediates estrogen receptor- $\alpha$-induced mesenchymal stem cell vascular endothelial growth factor production," Journal of Thoracic and Cardiovascular Surgery, vol. 138, no. 1, pp. 163.el-171.e1, 2009.

[32] J. E. Jung, H. G. Lee, I. H. Cho et al., "STAT3 is a potential modulator of HIF-1-mediated VEGF expression in human renal carcinoma cells," The FASEB Journal, vol. 19, no. 10, pp. 12961298, 2005.

[33] Q. Xu, J. Briggs, S. Park et al., “Targeting Stat3 blocks both HIF1 and VEGF expression induced by multiple oncogenic growth signaling pathways," Oncogene, vol. 24, no. 36, pp. 5552-5560, 2005. 

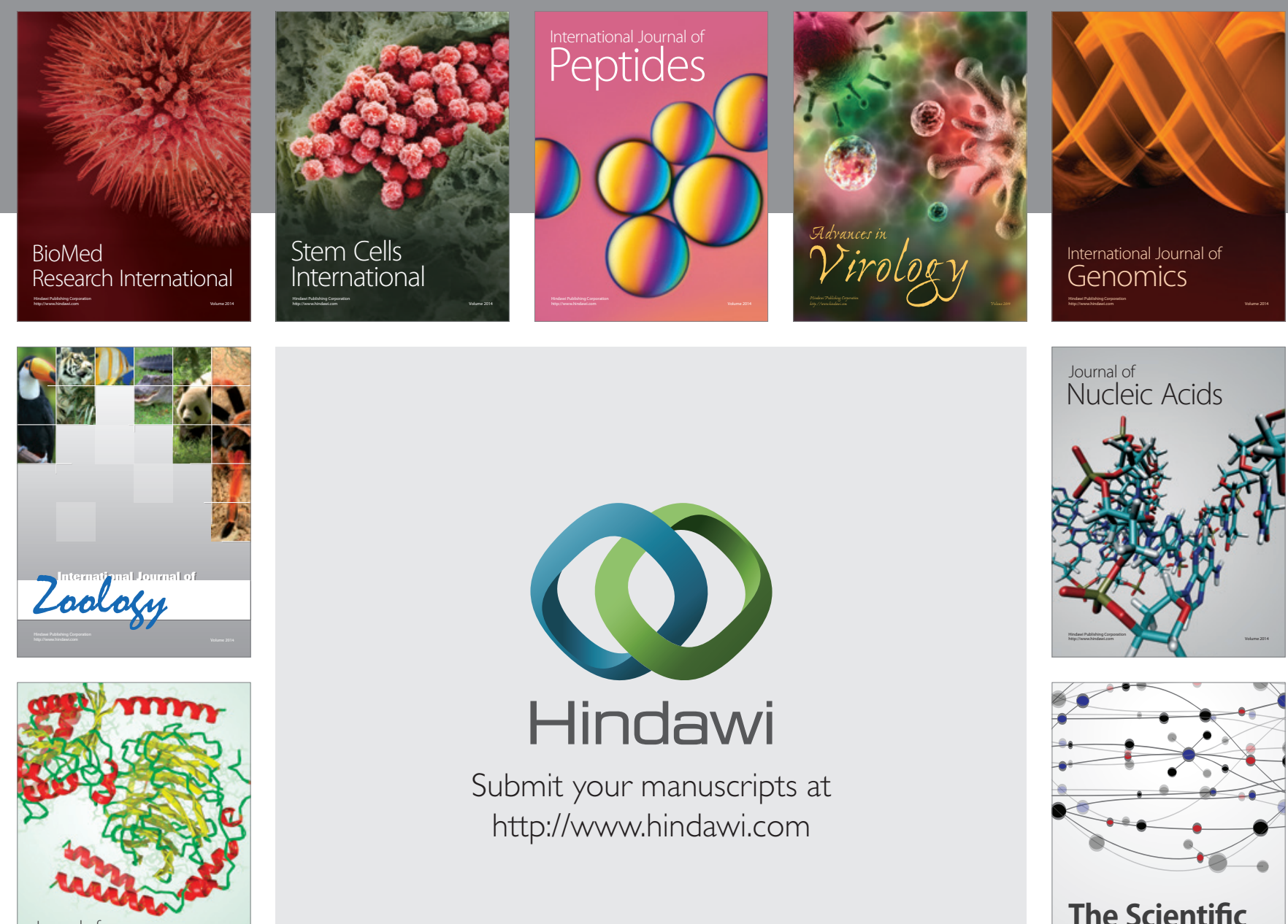

Submit your manuscripts at

http://www.hindawi.com

Journal of
Signal Transduction
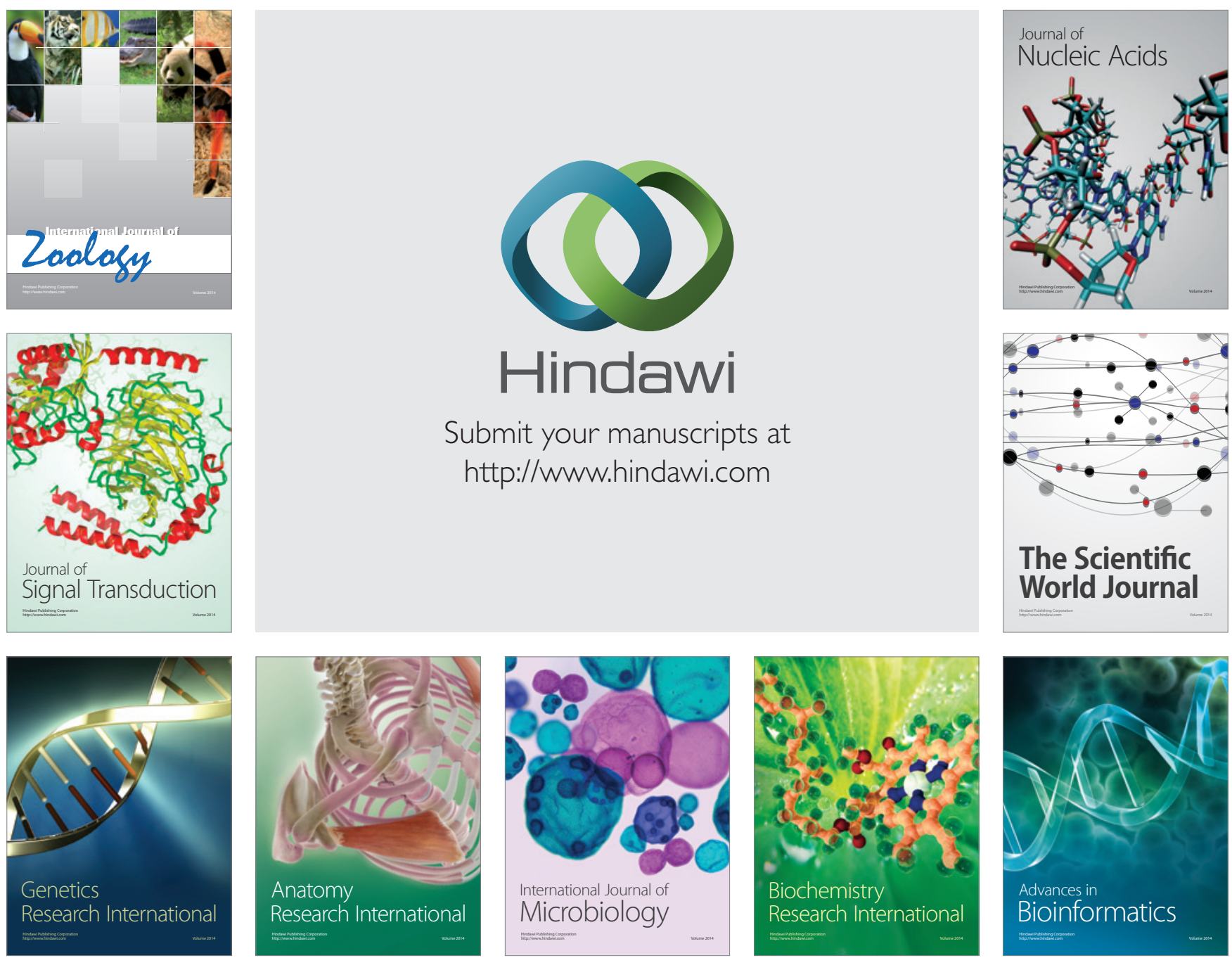

The Scientific World Journal
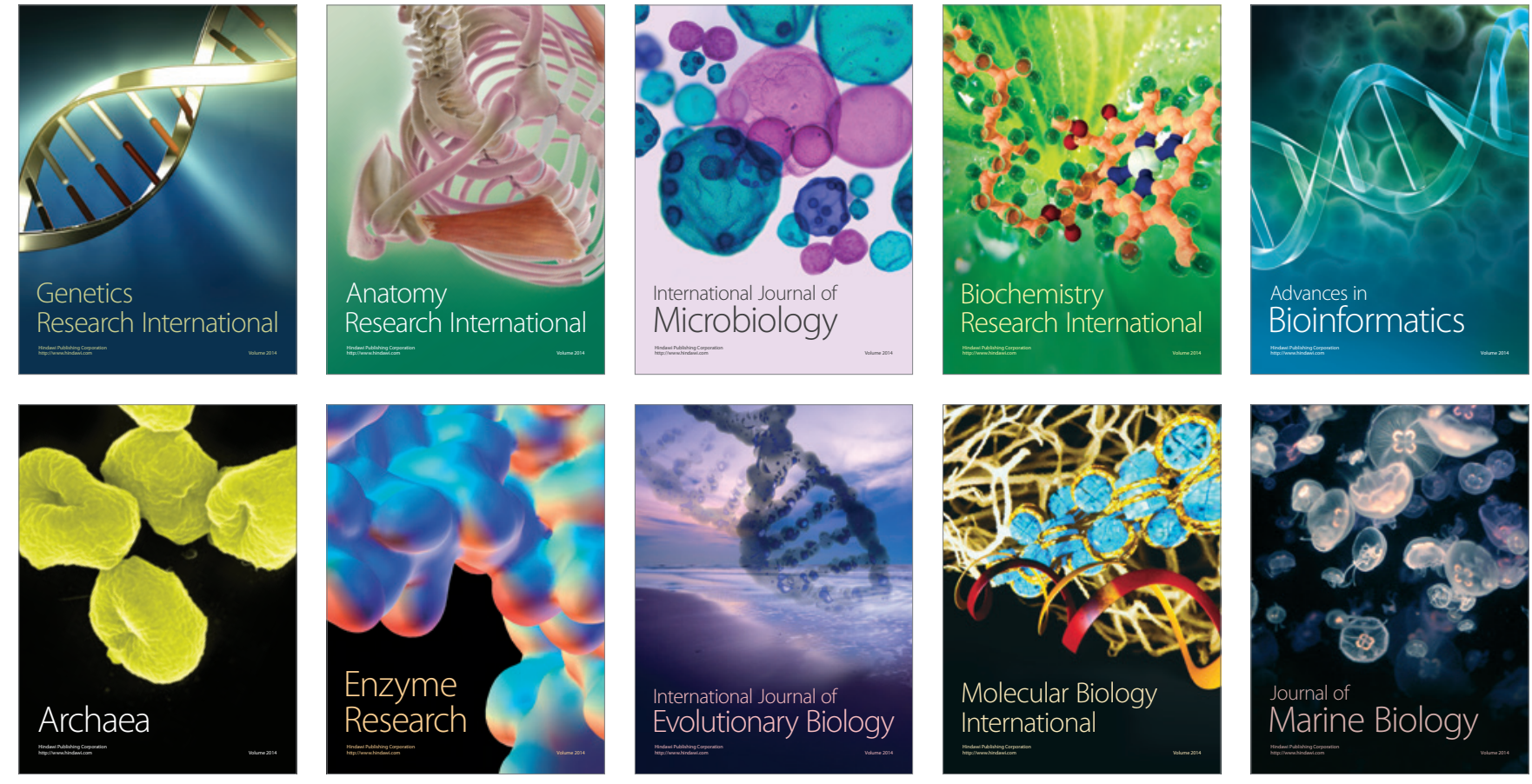